\title{
Sensitive Low Level Transistorized NMR Spectrometer Employing Frequency Modulation
}

\section{Authors: Fred Blankenburg, Roy Knispel, and V. Hugo Schmidt}

Copyright (1966) American Institute of Physics. This article may be downloaded for personal use only. Any other use requires prior permission of the author and the American Institute of Physics. The following article appeared in Review of Scientific Instruments and may be found at http://dx.doi.org/10.1063/1.1720396.

Blankenburg, Fred, Roy Knispel, and V. Hugo Schmidt. "Sensitive Low Level Transistorized NMR Spectrometer Employing Frequency Modulation." Review of Scientific Instruments 37, no. 8 (1966): 1020. doi:10.1063/1.1720396.

Made available through Montana State University's ScholarWorks scholarworks. montana.edu 


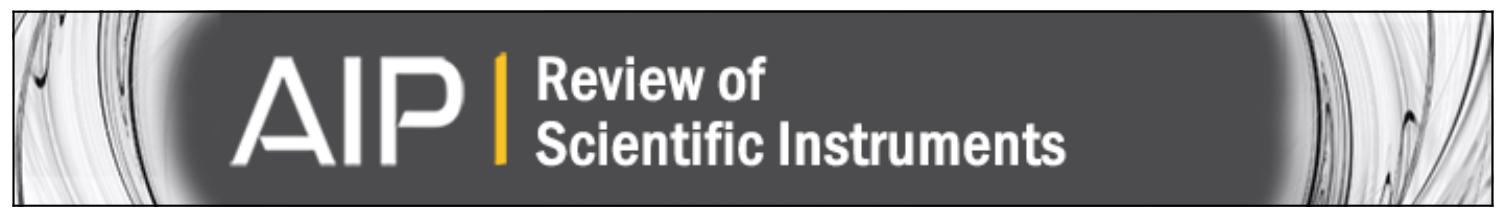

Sensitive Low Level Transistorized NMR Spectrometer Employing Frequency Modulation

Fred J. Blankenburg, Roy R. Knispel, and V. Hugo Schmidt

Citation: Review of Scientific Instruments 37, 1020 (1966); doi: 10.1063/1.1720396

View online: http://dx.doi.org/10.1063/1.1720396

View Table of Contents: http://scitation.aip.org/content/aip/journal/rsi/37/8?ver=pdfcov

Published by the AIP Publishing 
eter of the illuminated area remains constant for a wide range of electron flux densities which can be varied by the movement of the second condenser magnetic lens control.

In preliminary operation at $50000 \mathrm{~V}$, a negative bias was placed on the outermost tungsten circular grid in order to minimize primary reflections and secondary emissions of electrons impinging on the collector plate surface. It had been found on the experimental configuration that the difference in the recorded current with a 0 to $100 \mathrm{~V}$ bias was not detectable. This allowed bias as an experimental parameter to be eliminated. Next, the second condenser magnetic lens control is varied until the desired flux density is recorded on an electrometer. When the flux density is determined, the probe is rotated about its axis so as to orient the specimen at a prescribed angle to the beam. The alignment of beam, specimen, and densitometer is controlled by the movement of the two angular and lateral adjust micrometer screws. Specimens of various structures can be accommodated in the probe by the movement of the variable height adjustment screw.

The probe enables the user to subject bulk specimens to electron irradiation at controlled, stable energy levels over a wide range of electron flux densities. No alterations are necessary to existing Hitachi HU-11 and HU-11a electron microscope systems. The conversion from the material irradiating geometry to the normal microscopy and diffraction geometry is accomplished rapidly. The device can be of use in the study of electron radiation damage to crystals, electronic devices, thin metallic films, ceramics, and polymers in the low electron energy ranges and dose levels, similar to those of orbital missions of various earth satellites.

\title{
Sensitive Low Level Transistorized NMR Spectrometer Employing Frequency Modulation*
}

\author{
Fred J. Blankenburg, Roy R. Knisped, $\dagger$ and V. Hugo Schmidt \\ Department of Physics, Montana State Universily, Bozeman, Montana 59715
}

(Received 10 February 1966; and in final form, 7 April 1966)

\begin{abstract}
A transistorized NMR spectrometer has been developed which affords sensitivity comparable to that of the best vacuum tube spectrometers. This spectrometer has operated with sample coil voltages below $10 \mathrm{mV}$ peak-to-peak. Frequency modulation is used to facilitate modulation at frequencies high enough to minimize effects of transistor $1 / f$ noise.
\end{abstract}

\section{INTRODUCTION}

W E describe here a sensitive transistorized NMR spectrometer in which the frequency is modulated by means of a voltage sensitive capacitor. Transistorized circuits offer the advantages of simplicity, small space requirements, minimization of microphonics, and ability to operate in high magnetic fields. They require little power and can be battery operated, eliminating problems connected with line voltage fluctuations and ground loops through external filament and $\mathrm{B}^{+}$supplies. Frequency modulation eliminates need of an audio power amplifier or modulation coils and allows easier variation of the modulation frequency. The technique of modulation by means of a semiconductor diode especially designed for large variation of capacity with applied voltage was applied to NMR spectroscopy by Jennings and Tanttila, ${ }^{1}$ and was used with a transistorized spectrometer by Fric and Hahn. ${ }^{2}$ Several

\footnotetext{
* Supported in part by NIH under Grant GM-12383.

+ NASA Predoctoral Fellow.

D. A. Jennings and W, H. Tanttila, Rev. Sci. Instr. 30, 137 (1959).

2 C. Fric and H. Hahn, Compt. Rend. 250, 1471 (1960).
}

transistorized NMR circuits which have been described previously ${ }^{2-4}$ are variations of the marginal oscillator circuit. ${ }^{5}$ Transistorized versions of the autodyne circuit ${ }^{6}$ and the simple absorption circuit ${ }^{7}$ have also been described. Robinson ${ }^{8}$ has designed a transistorized magnetic field probe using a limited oscillator, and has found its signalto-noise ratio for a given sample to be about half that of a good vacuum tube spectrometer. Donnally and Sanders ${ }^{3}$ have obtained similar sensitivity with their transistorized marginal oscillator circuit. Most of the previously described transistorized circuits are used as magnetic field probes, and none has maximum possible sensitivity as the primary design consideration. Our spectrometer is used primarily to observe deuterons in hydrogen bonded crystals, and the

\footnotetext{
${ }^{3}$ B. Donnally and T. M. Sanders, Jr., Rev. Sci. Instr. 31, 977 $(1960)$.

${ }_{4}^{4}$ R. L. Garwin, A. M. Patlach, and H. A. Reich, Rev. Sci. Instr. 30, 79 (1959); J. R. Singer and S. D. Johnson, ibid. 30, 92 (1959); and W. L. Pierce and J. C. Hicks, ibid. 36, 202 (1965).

${ }^{5}$ R. V. Pound, Progr. Nucl. Phys. 2, 21 (1952).

${ }^{-}$F. Bruin and P. C. Van Soest, Rev. Sci. Instr. 31, 909 (1960)

7 R. L. Garwin and A. M. Patlach, Rev. Sci. Instr. 36, 741 (1965).

${ }^{8}$ F. N. H. Robinson, J. Sci. Instr, 42, 653 (1965).
} 


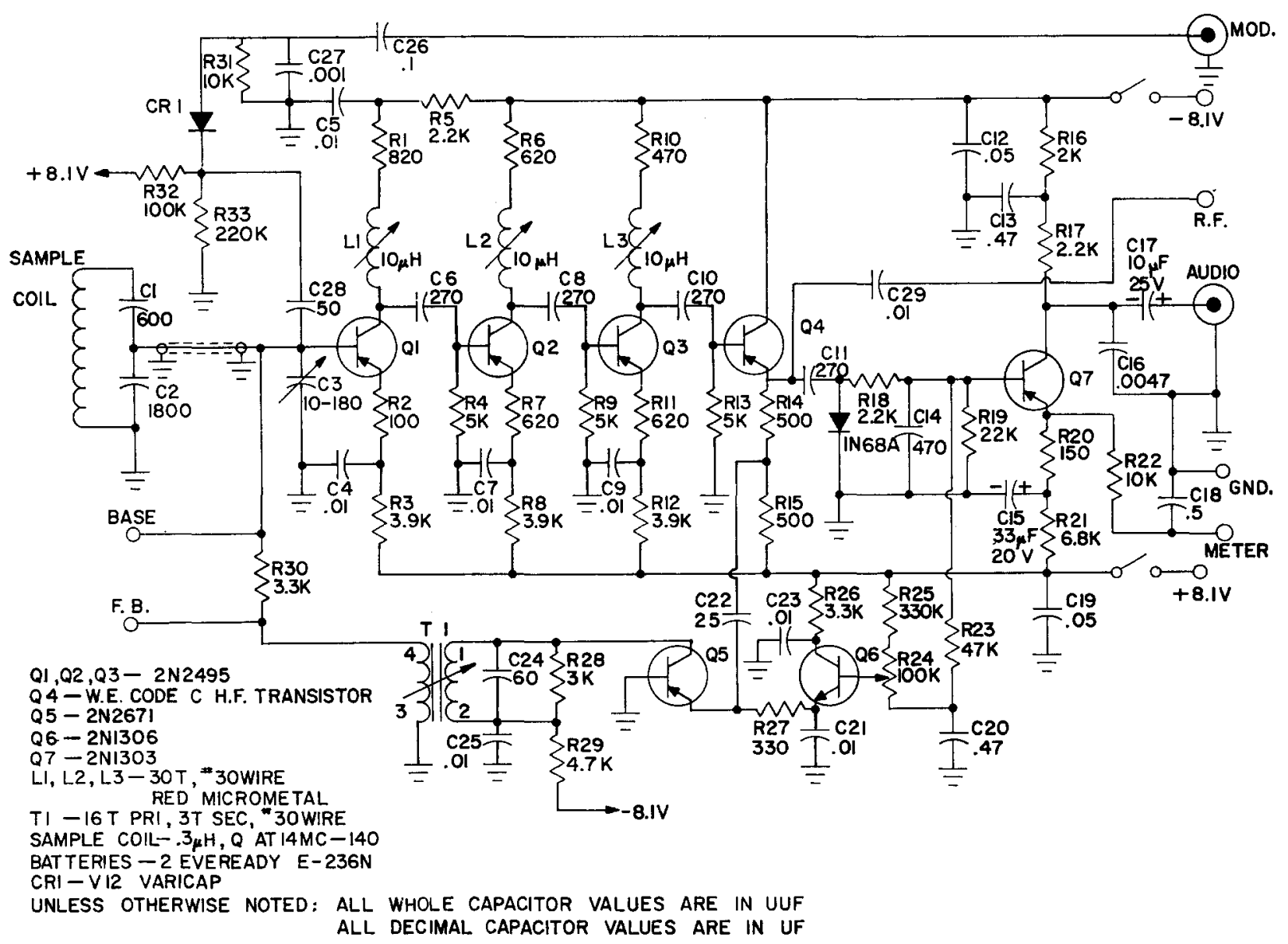

FIG. 1. Spectrometer circuit diagram.

small deuteron magnetic moment and the long spin-lattice relaxation time require maximum possible sensitivity and operation at low rf levels.

\section{CIRCUIT DESCRIPTION}

The spectrometer circuit is shown in Fig. 1. Basically it consists of a sample tank circuit feeding a three stage broad band rf amplifier followed by an audio stage, with the level of rf feedback to the tank circuit controlled by an automatic gain control (agc) circuit. The spectrometer is compact and self-contained, the only external connections being the frequency modulation input and the audio output cables. We obtain modulation voltage from the reference output of a Princeton Applied Research model JB-5 lock-in amplifier. The spectrometer output is fed to the signal input of this amplifier, and the amplifier output is displayed on a chart recorder.

The split capacity $\mathrm{C} 1$ and $\mathrm{C} 2$ across the sample coil is used to transform the relatively low impedance of Q1 up to a value which does not appreciably lower the $Q$ of the sample tank. The sample tank is connected to the rest of the circuit by a rigid coaxial cable $50 \mathrm{~cm}$ long consisting of a $2 \mathrm{~mm}$ diam copper wire inside a $35 \mathrm{~mm}$ diam aluminum tube. Cable losses cause no difficulty at our operating frequency of $14 \mathrm{Mc}$ because the capacity of the tuning capacitor C3 is relatively small. Frequency modulation is provided by the semiconductor variable capacitor CR1.

The provision of considerable gain in the rf amplifier takes advantage of the fact that transistor noise figure is lowest in the rf region. The transistors Q1, Q2, and Q3 are Amperex 2N2495; this type was selected because of low noise figure and low input and output capacitance. The noise from Q1 is reduced by keeping emitter resistance $\mathrm{R} 2$ small to increase the gain, and by reducing the collector voltage. The amplifier gain is determined by the values chosen for emitter resistors $\mathrm{R} 7$ and $\mathrm{R} 11$. The peaking coils L1, L2, and L3 increase gain and reduce phase shift, and are adjusted to flatten gain over a fairly wide band.

For the emitter follower Q4 we used a transistor from a Western Electric semiconductor kit, but any good high frequency transistor that can tolerate a constant emitter current of 10 or $12 \mathrm{~mA}$ can serve. The emitter follower drives the diode detector, which is followed by a single audio amplifier stage $Q 7$. The emitter of $Q 7$ is connected through a suitable resistor to a $100 \mu \mathrm{A}$ movement meter which indicates the rf level. 
The rf feedback is taken from the emitter follower $Q 4$ through an attenuation and phase shift network to Q5 and variable transformer $T 1$, and is fed to the sample tank through R30. The transistor Q5 is the nonlinear element which controls oscillation amplitude. Its operating point is determined by an agc circuit which includes the rf level control R24.

The modulation of the sample tank circuit capacity causes amplitude as well as frequency modulation even in the absence of a nuclear signal. To prevent overloading of the lock-in amplifier this amplitude modulation must be minimized by adjusting T1. If other parts of the circuit had no effect, tuning the feedback tank to the sample tank frequency would eliminate this amplitude modulation.

For best sensitivity the feedback current must be in phase with the base voltage of Q1. The negative phase shift in the rf amplifier is approximately compensated by $\mathrm{C} 22$, and remaining phase shift can be cancelled by adjusting the peaking coils. The correct phase gives maximum voltage ratio $\mathrm{BASE} / \mathrm{FB}$; voltages at these test points must be measured with high impedance $(<10 \mathrm{pF})$ probes. The relative phases at these test points can be observed directly with a good dual trace rf oscilloscope triggered from the rf test point. The adjustments for feedback phase and for amplitude modulation minimization unfortunately are not independent.

This circuit is built on a $10 \times 12.7 \mathrm{~cm}$ piece of Vector board, with a heavy ground bus along the center of the board. The grounds for each stage were kept short and brought to one point to minimize interaction between stages. The circuit, including its mercury batteries, is enclosed in a $10 \times 12.7 \times 15.2 \mathrm{~cm}$ aluminum case, with the rf level meter mounted on top.

\section{SENSITIVITY}

The oscillator equivalent circuit is shown in Fig. 2, with the sample tank circuit transformed to an equivalent parallel tank at the base of Q1. The variation in nuclear absorption at resonance due to the frequency modulation adds to the mean tank conductance $G$ a variation $G_{s}$ with time dependence $\exp \left(i \omega_{m} l\right)$, giving total tank conductance $G_{i}=G+G_{s}$. This variation adds a signal $V_{s}$ with similar time dependence to the carrier, which has mean amplitude $V$. The noise current generator $I_{n}$ adds a noise voltage $V_{n}$,

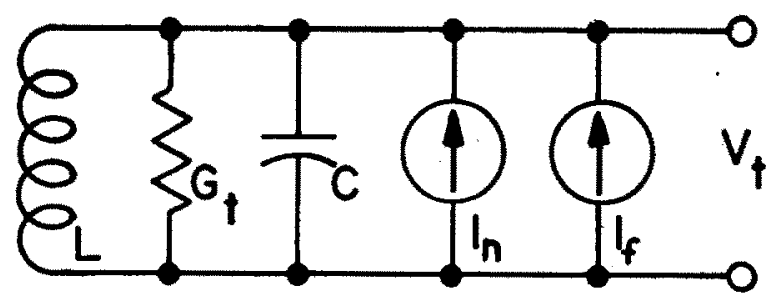

Fro. 2. Oscillator equivalent circuit. giving amplitude $V_{t}=V+V_{s}+V_{n}$ for the peak rf voltage at the base of Q1.

The effect of feedback is represented by a current generator $I_{f}$ because the feedback resistance R30 is much larger than $1 / G$. The nonlinearity of the feedback circuit gives instantaneous feedback current of

$$
i_{f}=\left(G_{0}-G_{1} V_{t} \sin \omega t-G_{2} V_{t}^{2} \sin ^{2} \omega t-\cdots\right) V_{t} \sin \omega t
$$

for instantaneous tank voltage $V_{t}$ sinwt. Ignoring terms beyond second order in $i_{f}$ and first order in $I_{f}$, the peak value of the component at frequency $\omega$ is

$$
\begin{aligned}
& I_{f}=\left(G_{0}-\frac{3}{4} G_{2} V_{i}^{2}\right) V_{t} \\
& =\bar{I}_{f}+\left(G_{0}-9 G_{2} V^{2} / 4\right)\left(V_{s}+V_{n}\right) \\
& \equiv \bar{I}_{f}+G^{\prime}\left(V_{s}+V_{n}\right) \text {; }
\end{aligned}
$$

The small magnitude and random phase of the noise current $I_{n}$ prevent it from contributing to the mean value of the current flowing in $G_{t}$. The mean value of the peak tank voltage is found from Eq. (3) to be

$$
V=\left[\frac{4}{3}\left(G_{0}-G\right) / G_{2}\right]^{\frac{1}{2}} .
$$

The magnitudes and phases of $V_{s}$ and $V_{n}$ are found by setting the rate of change of the tank energy equal to the power fed into the tank by the current generators less the power absorbed in the tank,

$$
d / d t\left(\frac{1}{2} C V_{t}^{2}\right)=\frac{1}{2} G_{t}^{-1}\left(I_{f}+I_{n}\right)^{2}-\frac{1}{2} G_{t} V_{t}{ }^{2} .
$$

Using the relation $\bar{I}_{f}=G V$, dividing through by $V$, and keeping terms to first order in $G_{s}, V_{s}, V_{n}$, and $I_{n}$, this equation becomes

$$
C\left(i \omega_{m} V_{s}+d V_{n} / d t\right)=\left(G^{\prime}-G\right)\left(V_{s}+V_{n}\right)+I_{n}-G_{s} V .
$$

This equation comprises two independent equations containing signal and noise terms, respectively.

The equation containing the signal terms yields

$$
V_{s}=-\frac{G_{s} V}{\frac{3}{2} G_{2} V^{2}+i \omega_{m} C}=-\frac{G_{s} V G^{-1}}{\frac{3}{2} G_{2} V^{2} G^{-1}+i Q \omega_{m} \omega^{-1}},
$$

where the relations $G-G^{\prime}=\frac{3}{2} G_{2} V^{2}$ [from Eqs. (2) and (3)] and $Q=\omega C / G$ have been used. The imaginary term describes a phase shift in $V_{s}$ which occurs for $\omega_{m}$ so high that the tank voltage cannot follow the modulation of $G$. This phase shift sets in at lower modulation frequencies as the rf level $V$ is reduced. For small $\omega_{m}$ the signal $V_{\mathrm{z}}$ is inversely proportional to the $\mathrm{rf}$ level $V$ in the absence of nuclear saturation.

For narrow band detection as obtained with a lock-in amplifier, only noise near $\omega_{m}$ in the spectrometer output need be considered. Because audio frequencies are strongly attenuated in the feedback loop, the generator $I_{n}$ represents rf noise which modulates the carrier amplitude at frequencies near $\omega_{m}$. Then $d V_{n} / d t$ in Eq. (6) can be replaced 
by $i \omega_{m} V_{n}$, and the noise terms in this equation give

$$
V_{n}=\frac{I_{n}}{\frac{3}{2} G_{2} V^{2}+i \omega_{m} C}=\frac{I_{n} G^{-1}}{\frac{3}{2} G_{2} V^{2} G^{-1}+i Q \omega_{m} \omega^{-1}} .
$$

The current generator of rms value

$$
\left(I_{n}\right)_{\mathrm{rms}}=(4 k T G F B)^{\frac{1}{2}}
$$

represents the combined effect of thermal noise in $G$ and the noise figure $F$ of the feedback loop. The bandwidth $B$ is determined by the lock-in amplifier. Dividing Eq. (7) by $\mathrm{Eq}$. (8) gives signal-to-noise ratio at the spectrometer input of

$$
\frac{V_{s}}{V_{n}}=\frac{G_{s} V_{\mathrm{rms}}}{\left(I_{n}\right)_{\mathrm{rms}}}=\frac{G_{s} V_{\mathrm{rms}}}{(4 k T G F B)^{\frac{1}{3}}}=\left(\frac{Q \omega C}{4 k T F B}\right)^{\frac{1}{2}} V_{\mathrm{rms}}\left(\frac{1}{Q}\right)_{s},
$$

where $(1 / Q)_{s}$ is the change in $1 / Q$ corresponding to $G_{s}$.

The foregoing analysis applies also to the marginal oscillator circuit, which has the same simplified equivalent circuit of Fig. 2. Actually the present circuit could be regarded as a marginal oscillator with an unusually long feedback path. The signal-to-noise ratio of $\mathrm{Eq} .(10)$ is the same as found by Robinson ${ }^{9}$ for his limited oscillator circuit. Robinson also analyzed the marginal oscillator circuit and obtained the same expression for signal voltage as given by Eq. (7) in the limit of low modulation frequency, but his expression for noise voltage in this limit is greater by a factor of 2 than the expression in Eq. (8). Robinson informs us that this result is erroneous and arose from the use of a noise power spectrum which is not applicable so near the frequency of oscillation.

The preceding analysis can be applied to the Robinson limited oscillator circuit. The limiting eliminates signal and noise feedback, so $G^{\prime}=0$ in Eqs. (2) and (6). The effect on $V_{s}$ and $V_{n}$ in Eqs. (7) and (8) is to replace the term $\frac{3}{2} G_{2} V^{2} G^{-1}$ by 1 , so that the denominators become $1+i Q \omega_{m} \omega^{-1}$. The replaced term is much smaller than unity, so the limited oscillator has smaller input signal $V_{s}$ than the present circuit, but can be operated at higher modulation frequencies before the phase shift due to the imaginary term sets in.

\section{RESULTS}

This spectrometer was originally used with magnetic field modulation at $380 \mathrm{cps}$. The signal-to-noise ratio for ${ }^{7} \mathrm{Li}$ in a single crystal of $\mathrm{LiD}_{3}\left(\mathrm{SeO}_{3}\right)_{2}$ was about 30 , half as great as the value obtained with a vacuum tube Robin$\mathrm{son}^{8}$ spectrometer. Use of frequency modulation at 1500 cps brought the signal-to-noise ratio up to that of our Robinson spectrometer. Study of the spectrometer output using a Hewlett-Packard model $300 \mathrm{~A}$ harmonic wave analyzer showed that noise power decreases as $1 / f$ or faster, with the noise corner near $10 \mathrm{kc}$, while our vacuum tube Robinson circuit has its $1 / f$ noise corner near $200 \mathrm{cps}$.

\footnotetext{
F. N. H. Robinson, J. Sci. Instr. 36, 481 (1959).
}

The signal voltage was found to vary inversely with if level as predicted by Eq. (7). The modulation frequency at which signal phase shift sets in also varies as predicted by Eq. (7), $1500 \mathrm{cps}$ for peak-to-peak tank voltage of 20 $\mathrm{mV}, 5 \mathrm{kc}$ for $36 \mathrm{mV}$, and $10 \mathrm{kc}$ for $57 \mathrm{mV}$. The corresponding range of the term $\frac{3}{2} G_{2} V^{2} G^{-1}$ in Eqs. (7) and (8), which is a measure of the effective nonlinearity of the feedback transistor Q5, is from 0.02 to 0.15 . The phase shift onset for a given rf level could be pushed to higher modulation frequency at the cost of decreased signal gain by increasing the effective nonlinearity of Q5.

The circuit as shown in Fig. 1 can oscillate with sample tank peak-to-peak voltages down to $20 \mathrm{mV}$, but by using greater rf gain we have achieved oscillation with tank voltage well below $10 \mathrm{mV}$. The oscillator frequency varies by less than 1 part in $10^{3}$ over the entire range of rf level. No attempt has been made to improve the frequency stability. The effect of reducing the agc bypass capacitor $\mathrm{C} 20$ was investigated. A value of $250 \mathrm{pF}$, which allows negative feedback of all audio frequencies, reduced the signal somewhat and partially eliminated the reduction in signal with increasing rf level. The signal-to-noise ratio was not appreciably affected.

Deuteron resonances have been observed with this spectrometer in single crystals of $\mathrm{LiD}_{3}\left(\mathrm{SeO}_{3}\right)_{2}$ and $\mathrm{LiN}_{2} \mathrm{D}_{5} \mathrm{SO}_{4}$ having volumes 6 and $8 \mathrm{~cm}^{3}$, respectively. For the selenite crystal the signal-to-noise ratio of approximately 4 for the best lines was about the same as obtained with our Robinson spectrometer. In lithium hydrazinium sulfate the $\mathrm{ND}_{3}$ groups gave signal-to-noise ratio as high as 40 in some orientations.

\section{DISCUSSION}

This circuit, though basically a marginal oscillator, achieves greater stability and is capable of operation at lower sample tank rf level than the usual marginal oscillator circuit because the nonlinear element Q5 is at a point in the circuit where the rf level is high. Its advantage over the limited oscillator is greater signal gain for a given number of stages.

To achieve maximum sensitivity with this spectrometer as with others, it is necessary to maximize $Q$, minimize feedback loop noise figure $F$, and insure that the feedback current is in phase with the sample tank voltage. This circuit requires particular care in choice of a modulation frequency high enough to escape transistor $1 / f$ noise but low enough to avoid difficulty with the lock-in amplifier due to signal phase shift.

\section{ACKNOWLEDGMENTS}

The authors gratefully acknowledge helpful discussions with J. D. Rompel and N. A. Shyne of the Department of Electrical Engineering. 